\title{
Development of SAM over Different Phenomenological Models for Better Explanation of Elastic Scattering
}

\author{
Fahmida Sharmin 1, *, Md. Azizur Rahman² \\ ${ }^{1}$ Department of Natural Science, Stamford University Bangladesh, Dhaka, Bangladesh \\ ${ }^{2}$ Department of Physics, University of Dhaka, Dhaka, Bangladesh
}

Email address:

soni_bd@yahoo.com (F. Sharmin)

\section{To cite this article:}

Fahmida Sharmin, Md. Azizur Rahman. Development of SAM over Different Phenomenological Models for Better Explanation of Elastic Scattering. International Journal of High Energy Physics. Vol. 2, No. 2, 2015, pp. 22-26. doi: 10.11648/j.ijhep.20150202.11

\begin{abstract}
The information on nuclear bulk properties such as shapes, size and interaction radius can be obtained by studying the elastic scattering phenomena. Absorption is the process through which particles are removed from the entrance channel. In this present work we have discussed different models, found out their ambiguities and finally arrived at SAM, because of which elastic scattering problem becomes simple and experimental data can be described without any knowledge about absorption mechanism.
\end{abstract}

Keywords: Elastic Scattering, SAM, Strong Absorption Model

\section{Introduction}

Nucleus is a complicated, many body problems and a bound system of nucleons, with very short range interaction. Nucleons or other strongly interacting particles can induce a variety of nuclear reactions, whose diversity is due to the individual properties, relative motions, energies of the colliding particles and the target nuclei. Simple and fundamental laws are required in interpreting data to unravel the known properties of the nuclei and this enables us to predict the unknown properties also.

The scattering involving complex nuclei represents a complicated quantum mechanical many-body problem and it is difficult to correlate the experimental data directly with the properties of fundamental nuclear interactions. It is necessary to devise simpler methods (models) which serve as an intermediary between the data and basic nuclear theory. These methods make use of simplifying assumptions by which certain average features of the many-body problem are connected directly with measurable quantities.

In order to learn about the nuclear properties, we have to study the nuclear interactions. There are different types of nuclear reactions, such as elastic scattering, inelastic scattering, radiative capture, disintegration process, nuclear fissions, heavy-ion reactions etc. Different methods are used on this purpose.

The "deductive" method, where specific assumptions are made on average nuclear interaction potential and the angular distributions arising from this potential are compared with experiment. Scattering processes can be explained by two main phenomenological methods, they are:

a. The "inductive" method, where data are analyzed in terms of the asymptotic properties of the scattered wave functions i.e., determination of the complex nuclear phases.

b. These methods are known as "phenomenological" and "potential" methods,respectively.In"phenomenological" models, the nucleus is assumed "black" or highly opaque to certain incident partial waves. This assumption can be expressed directly in terms of the complex reflection coefficient $\eta_{l}$. In "potential model" absorption is described by adding an imaginary part to the average interaction potential.

The first phenomenological model was the diffraction theory of Bohr, Pierls and Placzek and Bethe ${ }^{[2]}$ for high energy neutron scattering. Due to the presence of Coulomb interaction in the case of charged particles a correction in the scattering were done by Akhiezer and Pomeranchuk ${ }^{[3]}$. Blair ${ }^{[4]}$ investigated the scattering of heavier charged particles by means of sharp cut-off model. Greider and Glassgold ${ }^{[5]}$ gave generalizations of this model for high energy neutron scattering and Mclntyre et al. ${ }^{[6,7]}$ gave for heavy charged 
particle scattering.

Here we present some description of these models and finally arrive at the strong absorption model of Frahn and Venter ${ }^{[1]}$.

\section{Akhiezer-Pomeranchuk-Blair (APB) Model (Sharp Cut-off Model)}

From the elastic scattering data, the behavior of the potential and of the optical model wave function in the interior region of nucleus cannot be determined. For this reason and because of the continuous and discrete ambiguities in the optical potential for complex projectile, an alternative approach to elastic scattering has been developed in terms of parametric reflection co-efficient. The simplest model is called the sharp cut-off model, which was proposed by Akhiezer and Pomeranchuk ${ }^{[3]}$ and Blair ${ }^{[4]}$, gave the first modern interpretation of the alpha particle scattering data on the basis of this model. They assumed that a charged incident particle will follow a well defined trajectory, because of the charge of the target and that of the alpha- particle. From the classical point of view the particle does not have enough energy to reach the nuclear surface.

In the presence of a Coulomb field, scattering of particles with masses $m_{1}$ and $m_{2}$ and charges $z_{1} e$ and $z_{2} e$ is characterized by the Coulomb parameter,

$$
n=\frac{m z_{1} z_{2} e^{2}}{\hbar^{2} k}
$$

Where, $m=\frac{m_{1} m_{2}}{m_{1}+m_{2}}$ is the reduced mass of the system and $k$ is related to the incident energy.

In the quantum mechanical description of charged particle scattering, the reflection co-efficients $\eta_{l}$ are factorized into a Coulomb part:

$$
n=\frac{m z_{1} z_{2} e^{2}}{\hbar^{2} k}
$$

and a nuclear part,

$$
\eta_{l=} \eta_{l} e^{-2 i \sigma_{l}}
$$

where, $\sigma_{l}$ is the Coulomb phase-shift.

We define $L-1 / 2$ as the nearest integer $\leq l^{\prime}$, in the sharp cut-off model.In the "black nucleus" picture all partial waves up to $L-1 / 2$ are absorbed and all partial waves with $l^{\prime}>L-1 / 2$ are scattered by the Coulomb field, so that

$$
\begin{gathered}
\eta_{l}=0, \quad l \leq L-\frac{1}{2} \\
\eta_{l}=1, \quad l>L-\frac{1}{2}
\end{gathered}
$$

The corresponding scattering amplitudes become,

$$
f(\theta)=f_{C}(\theta)+\frac{i}{2 k} \sum_{l=0}^{L-\frac{1}{2}}(2 l+1) e^{2 i \sigma_{l}} P_{l}(\cos \theta)
$$

where,

$$
f_{C}(\theta)=-\left(\frac{n}{2 k}\right)\left(\csc \frac{\theta}{2}\right)^{2} \exp \left[-2 i n \ln \left(\sin \frac{\theta}{2}\right)+2 i \sigma_{o}\right]
$$

is the Coulomb amplitude.

Using essentially the sharp cut-off assumptions and making approximations, AkhiezerPomerenchuk ${ }^{[3]}$ arrived at the following expressions:

$$
\begin{gathered}
f(\theta)=i k\left[\frac{R J_{1}(k R \theta)}{k \theta}+2 i n \frac{J_{0}(k R \theta)}{(k \theta)^{2}}\right], \quad \eta \ll 1 \\
f(\theta)=\frac{n}{2 k\left(\frac{\theta}{2}\right)^{2}} \exp \left[-2 i n \ln \frac{\theta}{2}+2 i \sigma_{0}\right], \eta \gg 1, \theta \ll \frac{2 n}{k R} \approx \theta_{C} \\
f(\theta)=i k \frac{R J_{1}(k R \theta)}{k \theta} \exp [2 \operatorname{in} \ln R], \eta \gg 1, \theta \gg \frac{2 n}{k R} \approx \theta_{C}
\end{gathered}
$$

These formulae are valid for small angle $\theta$ and small $\mathrm{L}$ such that the uncertainty principle $\Delta \mathrm{L} . \Delta \theta \leq 1$ is obeyed.

The elastic scattering of strongly interacting particles is thus characterized by the interplay of Coulomb and the diffraction effects. The APB model is quite successful in explaining the departure of the cross-section from the Coulomb cross-section down to about $0.1 \%$ of the same. The sharp cut-off approximations have difficulties and the theoretical cross-sections have oscillatory patterns not found in the experiment. Generally the agreement in the Coulomb region is good.

\section{McINTYRE's Model (Modified Cut-off Model)}

The main discrepancy of the APB model along with a possible minor error due to the semi- classical approximation comes from the sharp cut-off assumptions. This was realized by Blair ${ }^{[4]}$ who expected that the amplitude of the diffraction oscillations should be damped by relaxing the sharp cut-off conditions by a modified one. Since the nuclear surface is a finite zone of thickness'd' $(\sim 0.5 \mathrm{fm})$ over which nuclear density decreases from an approximately constant value in the interior to zero at the edge, so particles moving along classical orbits penetrating this diffuse region will be only partially absorbed. Denoting the change in the classical angular momentum parameter corresponding to the diffuseness 'd' by ' $\Delta$ ', we have

$$
\left(2 l^{\prime}+1\right) \Delta=2 k d(k R-n)
$$

again putting,

$$
\left[l^{\prime}\left(l^{\prime}+1\right)\right]^{1 / 2} \approx L+1 / 2
$$

And

$$
L+1 / 2=k R[1-(2 n / k R)]^{1 / 2}
$$

we obtain

$$
\Delta=k d\{1-(n / k R) \mid 1-(2 n / k R)\}^{-1 / 2}
$$

and for energies well above the Coulomb barrier ( $\mathrm{n} \gg 1$ ), $\Delta$ 
assumes the form

$$
\Delta \approx k d
$$

However a simple modification of the sharp cut-off assumption did not give much improvement. The first successful generalization was given by McIntyre et al. ${ }^{[6,7]}$. And these are as follows

- A gradual transition from complete absorption to pure Coulomb scattering is introduced by a smoothing function given by

$$
\eta_{1} \exp \left(-2 i \sigma_{l}\right)=\left[1+\exp \left(\mathrm{L}_{\mathrm{A}}-1 / \Delta_{\Delta_{A}}\right)\right]^{-1}
$$

Where, $l$ is a continuous variable, $\Delta_{\mathrm{A}}$ is the measure of the width of the transition region in $\mathrm{l}$ space and $\mathrm{L}_{\mathrm{A}}$ is not restricted to integer values.

- Despite the considerable improvements some discrepancies still remained, particularly for alpha scattering at higher energies. With increasing $\Delta_{\mathrm{A}}$, the oscillations are damped too strongly. The 'rise' also vanishes. A nuclear phase shift $\delta_{l}$ is introduced in addition to the Coulomb phase shift for partial waves near $\mathrm{L}_{\mathrm{A}}$ values through the relation

$$
\delta_{l}=\delta\left[1+\exp \left(\left(1-\mathrm{L}_{\mathrm{A}}\right) /_{\Delta_{\delta}}\right)\right]^{-1}
$$

The functional forms used for $\eta_{1}$ and $\delta_{1}$ have no theoretical basis. The differential scattering cross section $\sigma_{\mathrm{SC}}(\theta)$ is then related to the Rutherford scattering crosssection $\sigma_{\mathrm{R}}(\theta)$ by

$$
\begin{gathered}
{\left[\sigma_{S C}(\theta) / \sigma_{R}(\theta)\right]=\mid-i \exp \left[-i n \ln \left(\sin ^{2} \frac{\theta}{2}\right)\right]-\frac{1}{n} \sin ^{2} \frac{\theta}{2} \times} \\
\left.\sum_{l=0}^{\infty}(2 l+1)\left(1-\left|\eta_{1}\right|\right) \mathrm{e}^{2 \mathrm{i} \sigma_{l}} \mathrm{e}^{2 \mathrm{i}\left(\sigma_{\mathrm{l}-} \sigma_{0}\right)} \mathrm{P}_{\mathrm{l}}(\cos \theta)\right|^{2}
\end{gathered}
$$

The modified cut-off model gives excellent fits to many angular distributions of elastic scattering of alpha particles and heavy ions. But McIntyre's model suffers from the drawback of not being general. It is not suitable for scattering, reaction and polarization.

\section{PORTER'S Model}

To describe the interaction between nuclear particles, Porter ${ }^{[8]}$ assumed that the decrease in the observed intensity is due to the absorption of the particles from the incident channel. Over and above, it has been assumed that the trajectory of the incident particles will remain unaltered by the nuclear field of the target nucleus and will be a straight line inside the nucleus.

Observing a plot of the cross-section ratio $\sigma(E, \theta) / \sigma_{R}(E, \theta)$ for alpha particle scattered versus the apsidal distance, which yields an approximately universal function (Wegner plot), Porter assumed that

$$
\sigma(E, \theta)=\sigma_{R}(E, \theta) \tau
$$

Where $\tau$ is a transmission factor giving the fraction of the incident particles that emerge from the interaction region. For strong absorption, $\tau$ is given by

$$
\tau=\exp \left[-\frac{2 R}{\lambda_{0}} P\left(\frac{D}{R}, \frac{a}{R}\right)\right]
$$

Where $\lambda_{0}$ is the mean free path for alpha particle in the nuclear interior, ' $a$ ' is the diffuseness of the nuclear density.

The critical point in the Porter's model lies not so much in the classical calculation of $\tau$, but rather in the basic assumption. Making this assumption one may neglect the interference between the partial waves, which gives rise to the diffraction pattern in the angular distribution. In the limit of 'black nucleus' approximation and for the scattering of nuclear particles, this amounts of neglecting the contribution to the total cross-section.

\section{Optical Model}

The optical model is based on the assumption that the many body interaction can be approximated by a two body one. Here the target nucleus is represented by a potential well to represent various interactions of an incident nucleon with the target nucleus and this potential is complex in general. The real part of the potential corresponds to elastic scattering and the imaginary part of the potential represents the inelastic processes. The whole process is called the optical model, because it has analogy with light being refracted and reflected when impinging on some targets, hence the name. In the optical model, a medium is characterized by a refractive index and an absorption coefficient. For nuclear scattering problem one solves the Schrondinger equation:

$$
\nabla^{2} \psi+2 m / \hbar^{2}(E-V) \psi=0
$$

Where $\mathrm{m}$ is the reduced mass, $\mathrm{E}$ is the energy in the centre mass system and $\mathrm{V}$ is the interaction potential. The optical potential is conveniently written in the form

$$
V(r)=U(r) f(r)+i W g(r)
$$

The form factors $f(r)$ and $g(r)$ are usually chosen to have a maximum value of unity or nearly unity, so that $U$ and $W$ are the depth of the real and imaginary parts of the potential respectively.

The optical model has a number of parameters and these do not always assume unique values; the imaginary part of the optical potential is directly associated with the mean free path of the

projectile in the nuclear matter. It is possible to determine the values of these mean free paths for different projectiles. In fact, the interaction between complex nuclei is a many body problem and complex potential can be calculated, at least in principle, from given nucleon-nucleon forces.

The optical model is usually more successful for heavy and medium weight target nuclei than for light ones. The energy levels in heavier nuclei are much closer together, so that isolated resonances do not affect the interaction as much as they do for light nuclei. For very light nuclei exchange interaction can take place sufficiently frequently to render the 
optical model invalid except at small scattering angles. An important drawback of this model is that the analysis does not yield a unique optical potential.

\section{Strong Absorption Model}

From our earlier descriptions of the sharp cut off and modified cut-off models, it appears that although these models have been very successful in describing the elastic scattering of high energy neutrons and the intermediate energy charged particles, from a more general point of view, there are several features expected of a nuclear model; these are as follows:

\subsection{Unified Description}

Though the physical ideas and basic assumptions are sound, there is no direct connection between the formalisms described by sharp and modified cut-off models. A unified description is therefore needed to describe the scattering of both neutral and charged particles at intermediate and high energy regions.

\subsection{Shape Dependence}

The model of McIntyre et $\mathrm{al}^{[6,7]}$ uses a special analytical form of the smoothing function in $l$ space. Since this choice is rather arbitrary, one would like to know the effects of different shapes of $l$ in the transition region and to establish those features of the strong absorption models that are shape dependent.

\subsection{Analytical Treatment}

The Blair and McIntyre models have the disadvantages that results can be obtained only by numerical computation. This often obscures the dependence of cross-section features of certain physically meaningful parameter combinations and sometimes raises doubts whether the parameter values derived from an analysis are unique. Many angular distributions have to be calculated before a good fit to the data is obtained. Therefore it would be desirable to have a closed analytical formulation which is sufficiently general to explain all the features of SAM and also accurate enough to allow a quantitative analysis of the experimental data.

\subsection{Meaning of Parameters}

The physical significance of the phenomenological parameters, like $L, \Delta_{A}, \delta$, i.e. how they are related to quantities characterizing the average nuclear interaction such as radius $\mathrm{R}$, surface diffuseness $\mathrm{d}$, mean free path $\lambda$ and refractive index $\mathrm{n}$ are not obvious. The usual interpretation based on semi-classical arguments, is not clear and reliable. It may be possible to compare a general analytical formula obtainable from a many body treatment through the scattering amplitudes. Such comparison would result in quantitative relations between phenomenological models and interaction parameters.

\subsection{Scope of Model}

The early strong absorption models unlike the complex potential (optical) are not as flexible as to describe a considerable range of elastic and inelastic scattering phenomena. A generalized phenomenological model should be able to cover for instance, polarization, inelastic scattering via excitation of low lying collective states, quasi-elastic ( $p$, n) processes via iso-spin coupling, nuclear transfer reactions etc.

Considering all these features, Frahn and Venter have shown that, by a slightly different formulation and generalization of the basic assumptions of the form of the scattering function $\eta_{l}$ in $l$ space, all the requirements can be met. The strong absorption model (SAM) thus developed by Frahn and Venter ${ }^{[1]}$, has all the advantages of the earlier APB models but none of their drawbacks.

The strong absorption model is characterized by the following parameters:

1. The cut-off angular momentum, T,

2. The rounding parameter, $\Delta$,

3. The phase shift parameter $\mu_{1}$, and $\mu_{2}$,

4. The transparency parameter, $\varepsilon$,

5 . The symmetry parameter, $\rho$.

In the case of heavy ion-nucleus scattering the effect of parameters $\mu_{2}, \varepsilon$, and $\rho$ are insignificant. Hence we consider three parameters SAM with $\mathrm{T}, \Delta$ and $\mu$. The parameters $\mathrm{T}$ and $\Delta$ are respectively related to the interaction radius $\mathrm{R}$ and surface diffuseness d. Strictly speaking $\mu / 4 \Delta$ is related to the nuclear phase-shift. We have the semi classical formula for $\mathrm{T}$ and $\Delta$ as:

$$
T=k R(1-2 n / k R)^{1 / 2}
$$

and,

$$
\Delta=k d(1-n / k R)(1-2 n / k R)^{-1 / 2}
$$

where $\mathrm{n}$ and $\mathrm{k}$ are the wave number and the Coulomb parameter respectively.

The total reaction cross-section is given by

$$
\sigma_{r}=\frac{\pi T^{2}}{K^{2}}\left[1+\frac{2 \Delta}{T}+\frac{1}{3} \pi^{2}\left(\frac{\Delta}{T}\right)^{2}-\frac{1}{3}\left(\frac{\mu}{T}\right)^{2} \frac{\Delta}{T}\right]
$$

SAM formalism can be employed for the treatment of the inelastic scattering phenomena. The angular distributions for inelastic scattering have some features are closely analogous to those of the corresponding elastic scattering cross-sections at first noted by Blair. For instance, in the presence of strong Coulomb interaction, the inelastic scattering oscillations are smoothed out to the same extent as in elastic scattering.

This model can easily be extended the spin $1 / 2$ particles by different parameterization of $\eta_{l}^{+}$and $\eta_{l}^{-}$, where $\eta_{l}^{+}$and $\eta_{l}^{-}$ are the scattering functions for parallel and anti parallel spin sub-states respectively and $\eta_{l}=1 / 2\left(\eta_{l}^{+}+\eta_{l}^{-}\right)$

This has been accomplished by Frahn and Venter ${ }^{[1]}$. 


\section{Conclusion}

Finally from this present work we can say that SAM model gives a good description of elastic scattering of heavy ions which is in good agreement with other sophisticated methods such as optical model, A.P.B and C.C.B.A models. These models involve much more complex calculations for crosssection evaluation. SAM is thus a useful, easier, simple and alternate method for obtaining various information about nuclear properties. We can also say that an overall good description of the scattering of heavy ions is given by the three parameters of SAM of Frahn and Venter ${ }^{[1]}$.

\section{References}

[1] (a) W. E. Frahn and R.H. Venter, Ann. Phys (N. Y.) 24 (1963) 243. (b) W. E. Frahn, in fundamental in nuclear theory (IAEA) 1967, P-1
[2] Bohr, Pierles, Plazek and Beyhe et al, Phys. Rev. 57 (1940) 471.

[3] A. Akhiezer and I. Pomeranchuk, Journal Phys.(USSR) 9, 471 (1945).

[4] J. S. Blair, Phy. Rev. 95, 1218 (1945).

[5] K. R. Griender and A. E. Glassgold, Ann of Phys. 10, 100 (1960).

[6] J. A. McIntyre, S. D. Beeker and K. A. Wang and L. C. Beeker, Phys. Rev. 117, 1337(1960)

[7] J. A. McIntyre, S. D. Beeker and K. A. Wang, in Proc. Second conf. Reactions between complex nuclei. Gattisburgh, 180 (1960).

[8] G. Placzek and H. A. Bethe, Phys. Rev. A 57 (1940) 1075. 